\title{
Proliferaciones algales 2000-2001 en Bahía de Banderas, México según el sensor SeaWiFS
}

\author{
María C. Gómez-Villarreal ${ }^{1}$, Marcos D. Martínez-Gaxiola² \& José L. Peña-Manjarrez ${ }^{3}$ \\ 1. Centro de Estudios Tecnológicos del Mar No. 6, Coral No. 2, La Cruz de Huanacaxtle, Nayarit, 63732, México, Tel. \\ y Fax: (329) 29556 10; cetmar06_invest@sep.gob.mx \\ 2. Centro de Estudios Tecnológicos dē Mar No. 6, La Cruz de Huanacaxtle, Nayarit, México; \\ martinezgax@gmail.com \\ 3. Centro de Estudios Tecnológicos del Mar No. 11, Ensenada, Baja California, México; jopema01@yahoo.com.mx
}

Recibido 19-VIII-2007. Corregido 30-VI-2008. Aceptado 31-VII-2008.

\begin{abstract}
Algal blooms at Banderas Bay, México (2000-2001), from SeaWiFS-sensor-data. Algal blooms for the period of 2000 and 2001 at Banderas Bay, México, were analyzed from SeaWiFS-ocean-color-sensor derived information. These events were related with the maximum chlorophyll week anomalies (ASi; a proxy variable constructed for the analysis of chlorophyll temporal variation in the bay). The winter-spring blooms were multispecific, while the summer-fall blooms were monospecific. Two proposals are made: 1) Winter-spring blooms are strongly related with mesoescale processes, due to their apparent temporal synchrony with the high chlorophyll levels in the coastal region from Sinaloa to Jalisco states; 2) Cochlodinium polykrikoides (Margalef 1961) blooms during the summer-fall periods could be induced by local conditions and the influence of previous events on the ecosystem. Rev. Biol. Trop. 56 (4): 1653-1664. Epub 2008 December 12.
\end{abstract}

Key words: algal blooms, satellite remote sensing, SeaWiFS, chlorophyll, Banderas Bay, Cochlodinium polykrikoides.

En el año 2000 se monitoreó el primer caso de proliferación ("florecimiento") algal (PA o "FA") provocado por el dinoflagelado ictiotóxico Cochlodinium polykrikoides (Margalef 1961) en Bahía de Banderas, México (Cortés-Lara et al. 2004), aunque esos autores lo describieron como Cochlodinium catenatum (Okamura 1916). La proliferación se muestreó cada quince días en cuatro estaciones costeras del primero de agosto al doce de diciembre. La ubicación de esas estaciones respondió más a una estrategia de monitoreo sistemático frente a zonas de posibles descargas de desechos antropogénicos que a un plan de seguimiento de FA en la bahía. Tal situación implicó que la discusión que se hizo acerca de la extensión y duración del FA en la bahía se realizó desde una perspectiva de submuestreo; es decir, a partir de información derivada de estaciones y frecuencias de muestreo inadecuadas para cubrir eventos de esta naturaleza. Una alternativa para resolver el problema del submuestreo espacio-temporal en los océanos y en zonas costeras, es la utilización de información derivada de sensores remotos satelitales (SRS). El uso de esta herramienta para la detección y su potencial uso en la predicción de proliferaciones algales nocivas (FAN) se ha propuesto por diferentes autores, a partir de la base de datos proporcionada por el sensor de color del océano "Sea-viewing Wide Field of view Sensor" conocido como SeaWiFS (Steidinger y Haddad 1981, Cullen et al. 1997, Tester et al. 1998, Stumpf 2001). Por tanto, resulta necesario analizar las escalas espacial y temporal del proliferación reportado por Cortés-Lara et al. (2004) con el uso de información derivada de SeaWiFS y explorar la frecuencia e intensidad 
de las proliferaciones presentados en esta bahía, aprovechando la utilidad que ofrecen los sensores remotos de color del océano en el estudio de FA en el Pacífico tropical mexicano.

Las concentraciones de clorofila superficial derivada de los SRS (denominada de aquí en adelante como "clorofila satelital"), no discrimina por sí misma entre los grupos fitoplanctónicos que producen el FA, a menos que se conozca por muestreos in situ a las especies dominantes en una proliferación o que éstas posean características ópticas discriminantes (Cullen et al. 1997, Schoefield et al. 1999). Si bien se han desarrollado algoritmos para la detección de grupos fitoplanctónicos específicos a partir de SRS (Brown y Yoder 1994, Subramaniam et al. 1999, Stumpf 2001, Canizzaro et al. 2002, Tomlinson et al. 2004) persisten problemas metodológicos y aún existen muchos grupos no estudiados desde tal perspectiva. La mayor parte de los algoritmos desarrollados en este sentido están limitados a determinadas condiciones regionales $\mathrm{y}$, por tanto, a condiciones oceanográficas específicas. Por ejemplo, proliferaciones en océano abierto de la cianobacteria Trichodesmium (Subramaniam et al. 2002) y cocolitofóridos (Brown y Yoder 1994) pueden detectarse a partir de la modificación del espectro de reflectancia; los bajos índices de absorción específica de las diatomeas permiten su detección en proliferaciones dominadas por este grupo taxonómico (Sathyendranath et al. 2004); proliferaciones de dinoflagelados con altas concentraciones de micosporina pueden identificarse a partir de la baja reflectancia en las bandas ultravioleta del espectro (Kahru y Mitchell 1998). Sin embargo, es muy difícil utilizar estos métodos en regiones costeras donde la información óptica que recibe el sensor satelital se ve afectada por elementos no presentes en océano abierto, como sedimentos en suspensión; más aún, la coloración en la superficie marina durante un evento de proliferación algal no corresponde a las propiedades de absorción de ningún grupo fitoplanctónico en particular, sino a efectos ópticos provocados por la gran concentración de material celular cerca de la superficie que influye en los índices de esparcimiento de la luz, la disminución relativa del grado de absorción por parte del agua y el incremento de absorción por sustancias húmicas (Roesler et al. 2004; Dierssen et al. 2006).

Si se parte de la premisa de que los errores inherentes al trabajo con información satelital en cuerpos costeros se mantiene constante por épocas del año, y no se busca trabajar con concentraciones de clorofila sino con su variablidad espacial, es posible utilizar esta herramienta para describir la temporalidad de eventos de proliferaciones algales entendidos como periodos en los que se detectan los más altos niveles de clorofila satelital. Así, en este trabajo se utilizó información derivada del sensor SeaWiFS para construir una aproximación a las anomalías de clorofila satelital dentro de la Bahía de Banderas, México, con el objetivo de describir la extensión espacial y temporal de las proliferaciones algales ocurridas durante los años 2000 y 2001.

\section{MATERIALES Y MÉTODOS}

La Bahía de Banderas se encuentra localizada en la costa tropical del Pacífico mexicano $\left(20^{\circ} 24^{\prime} 19^{\prime \prime}-20^{\circ} 45^{\prime} 20^{\prime \prime} \mathrm{N}, 105^{\circ} 13^{\prime} 44^{\prime \prime}\right.$ $\left.105^{\circ} 41^{\prime} 43^{\prime \prime} \mathrm{O}\right)$ teniendo como límites naturales al norte a Punta de Mita, Nayarit, y al sur a Cabo Corrientes, Jalisco. Se realizaron muestreos mensuales de marzo a julio del 2000, quincenales de agosto a diciembre del 2000, y mensuales de enero a diciembre del 2001. Se tomaron muestras de agua de mar a 0,3 y $5 \mathrm{~m}$ de profundidad utilizando una botella Van-dorn, las cuales fueron preservadas con solución concentrada de lugol-acetato para la identificación y cuantificación de especies fitoplanctonicas en cuatro estaciones cercanas a la costa. La cuantificación se realizó mediante el método de Utermöhl (1958) para lo cual se sedimentaron $10 \mathrm{ml}$ de muestra, se dejaron en reposo durante 24 horas y posteriormente se hizo la lectura de la cámara completa a 40x utilizando un microscopio invertido marca Iroscope. Las densidades celulares de C. polykrikoides de agosto a diciembre del 2000 se promediaron para cada 
fecha de muestreo, tomando en cuenta las tres profundidades de las cuatro estaciones, estos promedios fueron utilizados para generar una serie de tiempo.

Se obtuvieron compuestos mensuales (enero de 1998 a diciembre de 2005) y compuestos semanales (enero de 2000 a diciembre de 2001) de clorofila satelital (imágenes hdf, nivel 3, resolución espacial aproximada de 9 $\mathrm{km}$ ) del sensor satelital de color del océano SeaWiFS, del servidor de distribución de datos de la NASA (http://oceancolor.gsfc.nasa.gov/ ftp.html). El procesamiento de la información derivada de estas imágenes se realizó con el software Windows Image Manager (www. wimsoft.com).

Para tener una idea de la extensión y periodicidad de los FA en las cercanías de la Bahía de Banderas, a partir de imágenes globales se recortaron compuestos mensuales de clorofila satelital de enero del 2000 a diciembre del 2001, desde las costas del estado de Sinaloa hasta el sur del estado de Jalisco, en el Pacífico tropical mexicano.

La clorofila satelital presenta alta interferencia por efecto de la costa, debido a esto se obtuvo una primera aproximación de la variabilidad de la clorofila satelital dentro de Bahía de Banderas a través de la construcción y uso del parámetro proxy Clorofila'. Este parámetro se construyó a partir de la mediana geográfica de la clorofila satelital obtenida de un área dentro de la Bahía de Banderas que evita la mayor parte de la señal de la costa (entre los $20^{\circ} 33^{\prime} 04^{\prime \prime}$ a $20^{\circ} 44^{\prime} 02^{\prime \prime} \mathrm{N}$ y $\operatorname{los} 105^{\circ} 23^{\prime} 13^{\prime \prime}$ a $105^{\circ} 43^{\prime} 48^{\prime \prime}$ O; Fig. 1).

Las premisas y supuestos implicados en este parámetro son:

1. El efecto de la costa sobre los niveles de clorofila satelital puede atenuarse eligiendo un área dentro de la bahía que excluya pixeles que mantienen altos valores de este parámetro en toda la serie temporal (Fig. 1).

2. El usar la mediana de los valores contenidos en el área seleccionada elimina el efecto de pixeles que posean valores extremos producto de la cercanía de la costa.
3. Los errores que aún conserva la mediana por efecto de los procesos costeros se consideran constantes en el tiempo, por lo que es factible usar y discutir su variabilidad temporal.

4. Al usar anomalías de Clorofila' en lugar de valores absolutos de este parámetro, se evitó la discusión referente a las concentraciones "reales" de pigmentos en la bahía y se clarifica su tendencia temporal.

Con los compuestos mensuales de 1998 a 2005 se generó una climatología mensual de Clorofila'. Esta climatología se usó para generar anomalías semanales de Clorofila' para los años 2000 y 2001 según la relación: $\mathrm{As}_{\mathrm{i}}=\mathrm{Xs}_{\mathrm{i}}$ $\mathrm{Xm}_{\mathrm{j}}$; donde $\mathrm{As}_{\mathrm{i}}$ son las anomalías de Clorofila' para cada semana $\left(\mathrm{s}_{\mathrm{i}}\right)$ del año, $\mathrm{Xs}_{\mathrm{i}}$ son los datos de Clorofila' para cada semana $\left(\mathrm{s}_{\mathrm{i}}\right)$ del año, y $\mathrm{Xm}_{\mathrm{j}}$ es la climatología de Clorofila' (19982005) para cada mes del año $\left(m_{j}\right)$, correspondiendo cada $\mathrm{Xm}_{\mathrm{j}}$ al mes que contiene a un grupo de $\mathrm{Xs}_{\mathrm{i}}$. Con esta información se generó una serie de tiempo con las $\mathrm{As}_{\mathrm{i}}$ para los años 2000 y 2001.

\section{RESULTADOS}

Las imágenes mensuales de clorofila satelital para las costas de Sinaloa a Jalisco mostraron que para ambos años (2000-2001) de febrero a mayo se presentaron los mayores niveles de clorofila satelital, mientras que de julio a octubre se registró la menor concentración, excepto en agosto del 2000 donde la clorofila satelital presentó un evidente incremento (Fig. 2). Durante el periodo de enero a mayo, en los dos años de estudio, se observó una aparente correspondencia entre los altos niveles de clorofila satelital detectados para la Bahía de Banderas y los altos niveles de clorofila satelital presentes en la región costera comprendida desde el norte del estado de Sinaloa hasta el sur del estado de Jalisco, lo que podría sugerir que las densidades fitoplanctónicas de la bahía respondiendo a fenómenos de mesoescala además de la influencia de forzamientos locales (Fig. 2). 


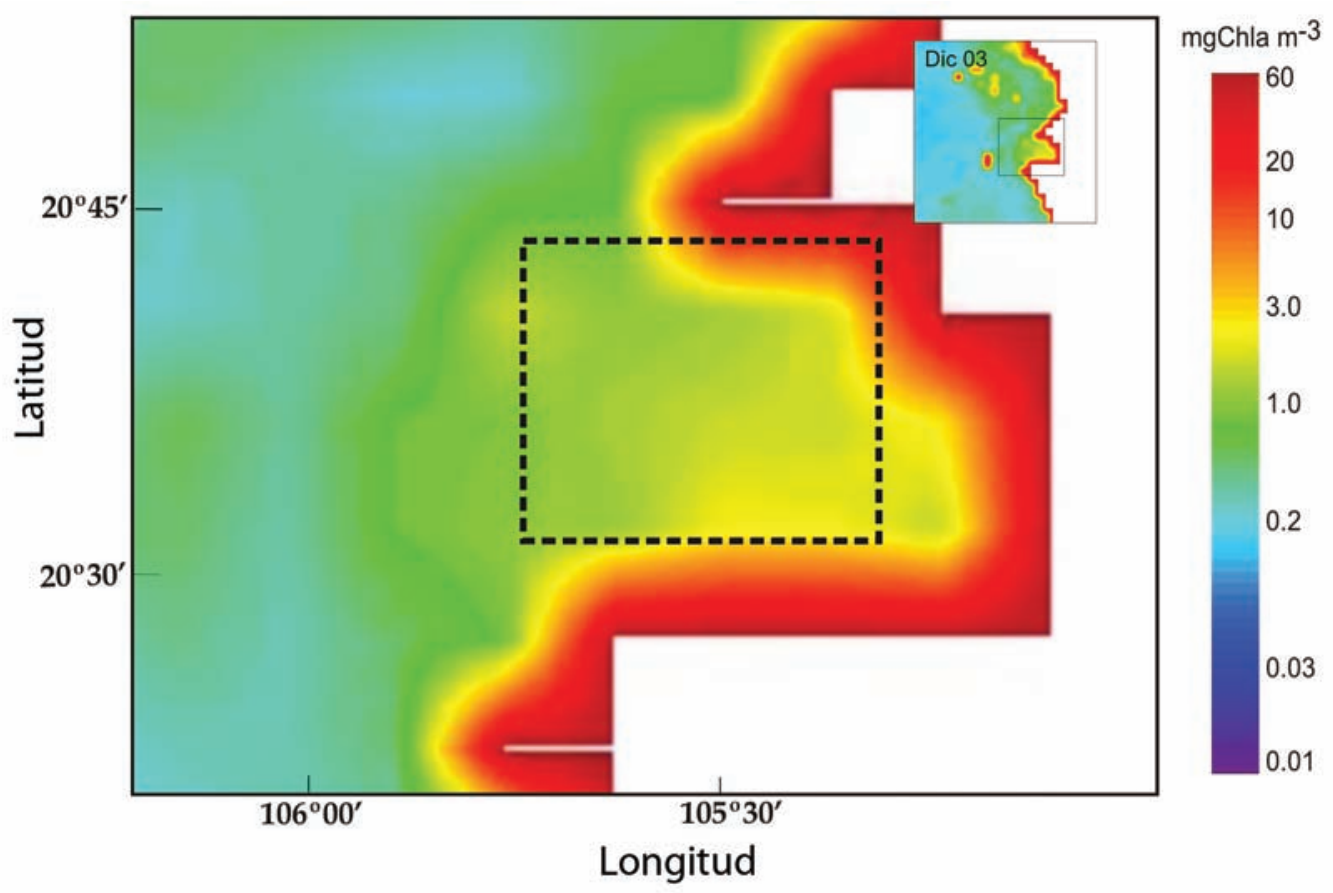

Fig. 1. Con líneas punteadas se delimita el área dentro de Bahía de Banderas utilizada para obtener el parámetro proxy Clorofila'. La imagen corresponde a un compuesto de clorofila satelital para la semana del 3 al 10 de diciembre del 2000, resolución espacial de 9 × $9 \mathrm{~km}$, de SeaWiFS. La sección blanca es la costa, a la que se encuentran adyacentes pixeles que poseen los valores más altos de "clorofila". La escala de color corresponde a la concentración de clorofila superficial determinada por los algoritmos correspondientes al sensor satelital (imágenes nivel 3).

Fig. 1. The area, inside Banderas Bay and delimited with a dotted line, is utilized to obtain the proxy parameter Clorofila'. The image corresponds to satellite chlorophyll for the week of December 3 to 10 of 2000, spatial resolution of $9 \times 9 \mathrm{~km}$, from SeaWiFS. The white section is the coast, to which pixels that posses the highest values of "chlorophyll" are adjacent. The color scale corresponds to the superficial chlorophyll concentration determined by the corresponding algorithms to the satellite sensor (level 3 images).

Por otra parte, si bien regionalmente las tendencias en los niveles de clorofila satelital son similares en los dos años revisados, dentro de la Bahía de Banderas la Clorofila' resultó muy distinta en estos periodos. En el 2000 predominaron las $\mathrm{As}_{\mathrm{i}}$ positivas durante la mayor parte del año, con los máximos niveles desde la última semana de marzo a la segunda semana de mayo; las máximas $\mathrm{As}_{\mathrm{i}}$ negativas, correspondientes a los valores más bajos de Clorofila', se presentaron en las cuatro semanas de diciembre. En tanto que en el periodo de toma de muestras en las cuatro estaciones de estudio de agosto a diciembre, correspondientes a la presencia de $C$. polykrikoides, las $\mathrm{As}_{\mathrm{i}}$ más altas se presentaron durante la última semana de septiembre y la tercer semana de noviembre (Fig. 3, 2000).

Los niveles de Clorofila' durante el 2001 en Bahía de Banderas fueron menores que los correspondientes al 2000. Las mayores $\mathrm{As}_{\mathrm{i}}$ positivas en el 2001 registraron valores equivalentes a la cuarta parte del nivel máximo registrado en el 2000, mientras que las $\mathrm{As}_{\mathrm{i}}$ negativas del 2001 fueron dos veces mayores que las del 2000. Las mayores anomalías de Clorofila' para el 2001 se presentaron durante febrero, julio y agosto; mientras que las anomalías 

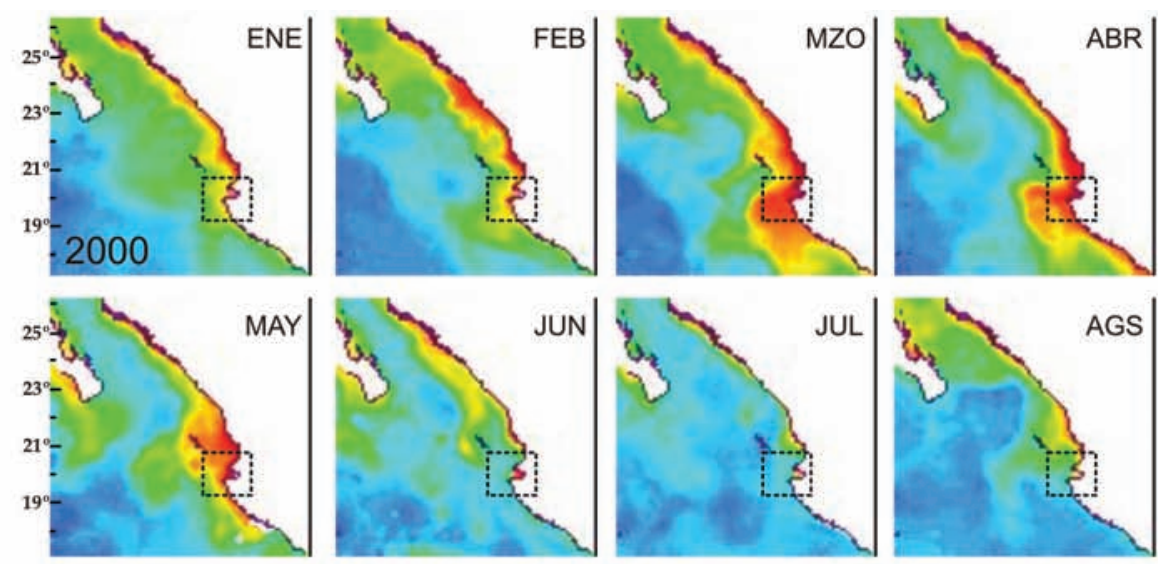

$\mathrm{mgChla} \mathrm{m}^{-3}$
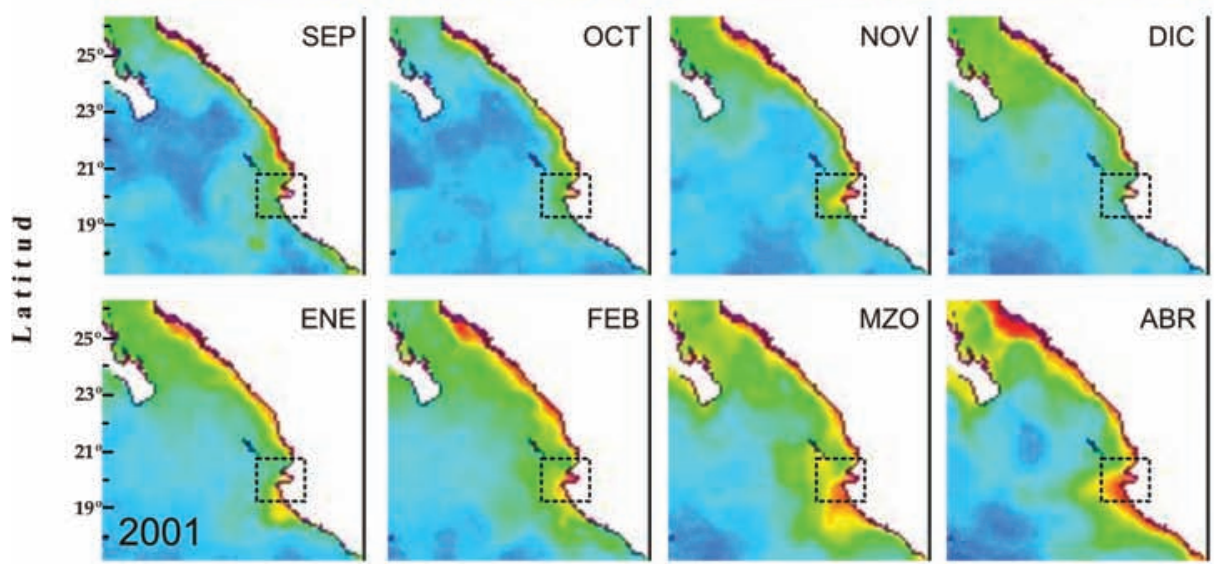

20
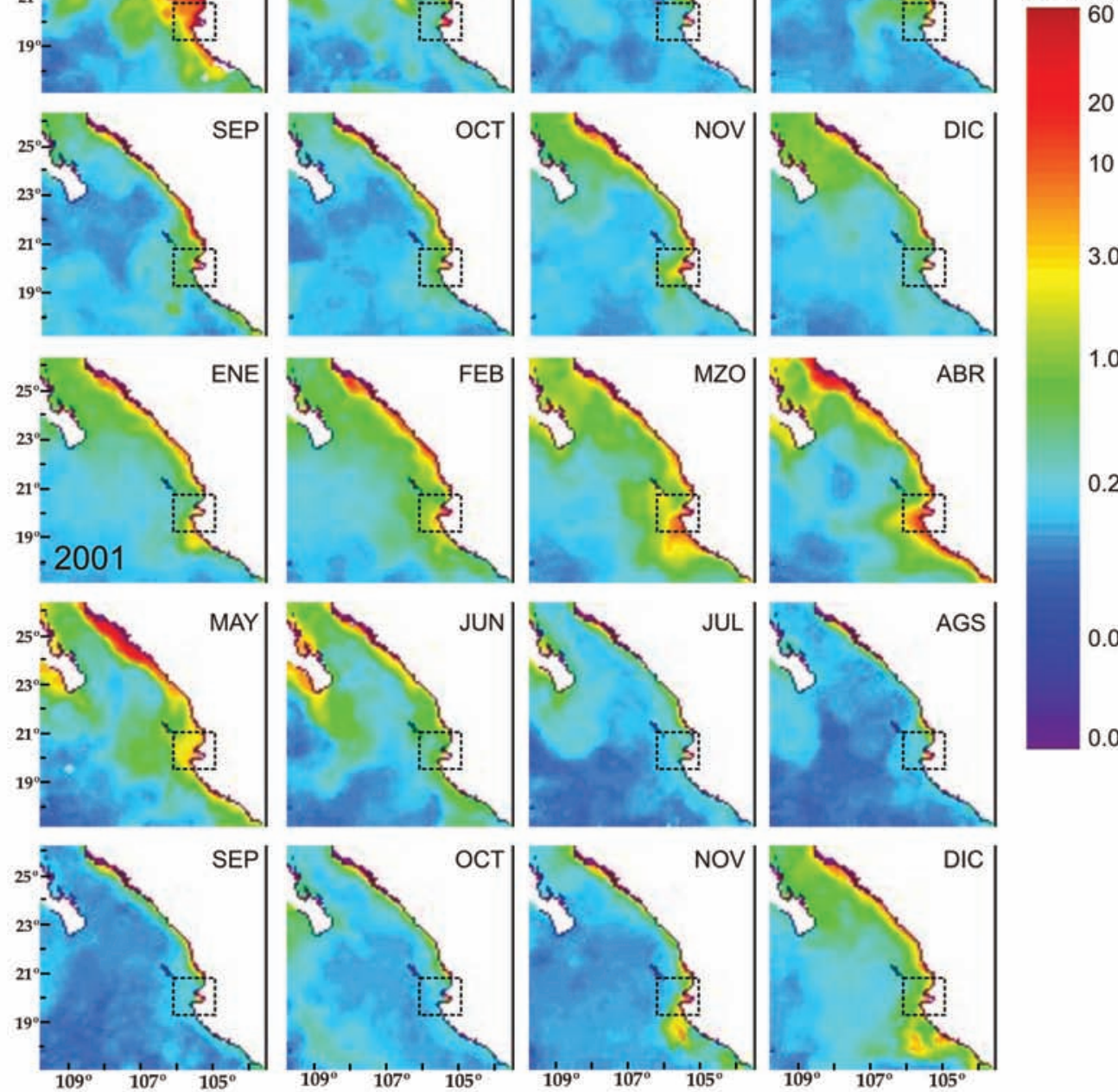

Longit ud

Fig. 2. Evolución de clorofila satelital en las costas de Sinaloa a Jalisco de enero de 2000 a diciembre de 2001. Bahía de Banderas se encuentra encerrada por un recuadro punteado. La escala de color corresponde a la concentración de clorofila superficial determinada por los algoritmos correspondientes al sensor satelital (imágenes nivel 3).

Fig. 2. Evolution of satellite chlorophyll in the coastal area from Sinaloa to Jalisco during January 2000 to December 2001. Banderas Bay is enclosed by a dotted box. The color scale corresponds to the surface chlorophyll concentration determined by the corresponding algorithms of the satellital sensor (level 3 images). 

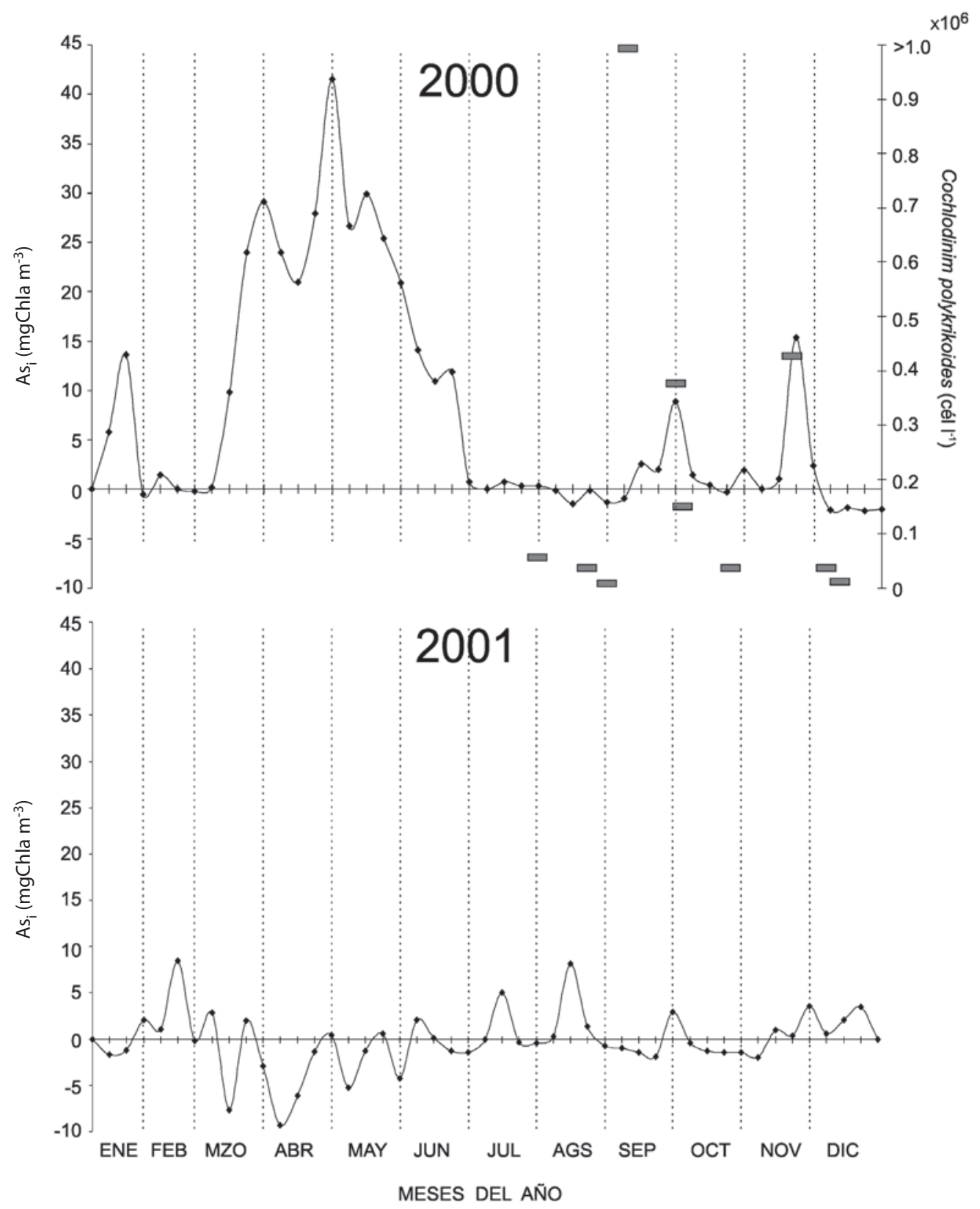

Fig. 3. Serie de tiempo de anomalías semanales de Clorofila' ( $\mathrm{As}_{\mathrm{i}}$, escala de la izquierda) en Bahía de Banderas, México, durante los años 2000 y 2001, calculadas como la diferencia entre la media semanal menos la media mensual de largo periodo (1997-2005). Los cuadros de tono gris en la gráfica del 2000 están referenciados en el eje de la derecha y señalan el promedio de las densidades celulares de C. polykrikoides considerando las tres profundidades de las cuatro estaciones de muestreo dentro de la bahía.

Fig. 3. Time series of weekly anomalies of Clorofila' (As, left scale) in Banderas Bay, Mexico, during the years 2000 and 2001, calculated as the difference between the weekly mean minus the monthly mean of long period (1997-2005). The gray boxes in the graph for 2000 are referred to in the right side axis and they show the average of the cell densities of $C$. polykrikoides considering the three depths in the four sampling stations inside the bay. 
negativas tuvieron sus máximos niveles durante marzo a mayo (Fig. 3, 2001).

Sólo se tiene la relación de especies que estaban presentes de marzo a mayo del 2000. Debido al enfoque del presente trabajo, se presentan a continuación los organismos que en forma relativa dominaron durante cada mes: marzo: Chaetoceros affinis (Lauder 1864), Chaetoceros decipiens (Cleve 1873); abril: Prorocentrum micans (Ehrenberg 1833), Dictyocha califórnica (Schrader et Murray 1985); mayo: P. micans, D. californica. En abril y mayo del 2000 las manchas de la proliferación algal registraron mayor extensión e intensidad (Fig. 3); en estos meses se observó que $P$. micans dominó la comunidad del fitoplancton.

Para efectos comparativos, se graficaron las densidades celulares promedio de $C$. polykrikoides de agosto a diciembre del 2000 en la misma Fig. 3 que muestra la serie temporal de $\mathrm{As}_{\mathrm{i}}$ para el 2000. Estas densidades celulares promedio oscilaron entre $7 \times 10^{3}$ y $1.2 \times 10^{6}$ cel $1^{-1}$, correspondientes a muestreos realizados en agosto y septiembre, respectivamente (Fig. 3, 2000). No se realizó la identificación del resto de organismos fitoplanctónicos presentes debido a la gran dominancia de C. polykrikoides en todas las muestras de agosto a diciembre de este año.

En los primeros tres meses del 2001 no se presentaron FA. En los meses siguientes se registró la presencia de las especies: abril: Gymnodinium catenatum (Graham 1943) , Myrioneta rubra (Lohmann 1908; Jankowski 1976); mayo: Ceratium furca (Ehrenberg; Claparede et Lachman 1859) y Ceratium balechii (Meave, Okolodkov et Zamudio 2003); junio: Navícula spp.; agosto a diciembre: $C$. polykrikoides. En estos últimos meses las densidades celulares promedio de C. polykrikoides

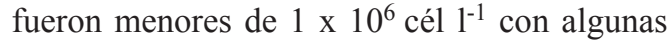
apariciones esporádicas, $\mathrm{y}$ abundancias muy bajas a partir de mayo de ese año.

\section{DISCUSIÓN}

El hecho de que en Bahía de Banderas prevalecieron $\mathrm{As}_{\mathrm{i}}$ positivas durante casi todo el año 2000 (Fig. 3) concuerda con las mayores tasas de producción primaria registradas dentro del periodo 1998-2005, tanto para el Pacífico tropical mexicano como para el Golfo de California (Martínez-Gaxiola et al. 2007), regiones oceanográficas que ejercen gran influencia en la bahía. Sosa-Ávalos et al. 2006, explican estos niveles de producción primaria en el Pacífico mexicano como resultado de una nutriclina más somera que aportó nutrientes a la zona eufótica de la región, condiciones oceanográficas asociadas al fenómeno La Niña 1999-2000 (Bograd y Lynn 2001, Carrasco y Quintana-Arena 2004).

Durante 1997-1998 se presentó el evento de El Niño, con un fuerte efecto en las tasas de producción primaria de la región (Bograd y Lynn 2001, Sosa-Ávalos et al. 2006), porque la termoclina es más profunda cerca de la costa y por tanto disminuye el ingreso de nutrientes hacia aguas superficiales, limitando la producción primaria (Fiedler et al. 1992). Es interesante plantear la posibilidad de que la alternancia El Niño-La Niña (1997-2000) hayan generado las condiciones en el ecosistema del Pacífico tropical mexicano que favorecieron la generación de las altas concentraciones de clorofila satelital detectadas en la región de Sinaloa a Jalisco (Fig. 2), en los altos niveles de Clorofila' en Bahía de Banderas durante la primera mitad del 2000 y hayan incidido en la proliferación de $C$. polykrikoides en la bahía a fines de este mismo año (Figs. 3 y 4).

Cortés-Lara et al. (2004) informaron que la duración de la proliferación de Cochlodinium catenatum fue de 18 semanas; sin embargo, este periodo se circunscribe únicamente al muestreo realizado en las cuatro estaciones cercanas a la costa de la bahía. Por ello, cabe la posibilidad de que este evento haya iniciado a finales de julio y simplemente no haya estado presente en las condiciones costeras en las que se realizaron los muestreos. Esta idea podría fortalecerse si las concentraciones de clorofila satelital para la región de Sinaloa a Jalisco de marzo a mayo del 2000 (Fig. 2) y las anomalías positivas de Clorofila' dentro de la Bahía de Banderas durante marzo a junio del mismo año 
(Fig. 3, 2000) se asociaran a la presencia de $C$. polykrikoides a partir de muestras in situ en la región central y en la boca de la bahía.

De haber sido así, las anomalías de Clorofila' durante la primera mitad del 2000 estarían indicando que en ese periodo se presentó un FA mucho más intenso que el estudiado in situ, que de junio a agosto se tuvo un periodo de relajamiento del evento y que existieron dos pulsos, uno a fines de septiembre y otro a fines de noviembre, mismos que coincidieron con las tomas de muestras (Fig. $3)$. Estas posibilidades no pueden ir más allá, debido a la ausencia de datos que demuestren

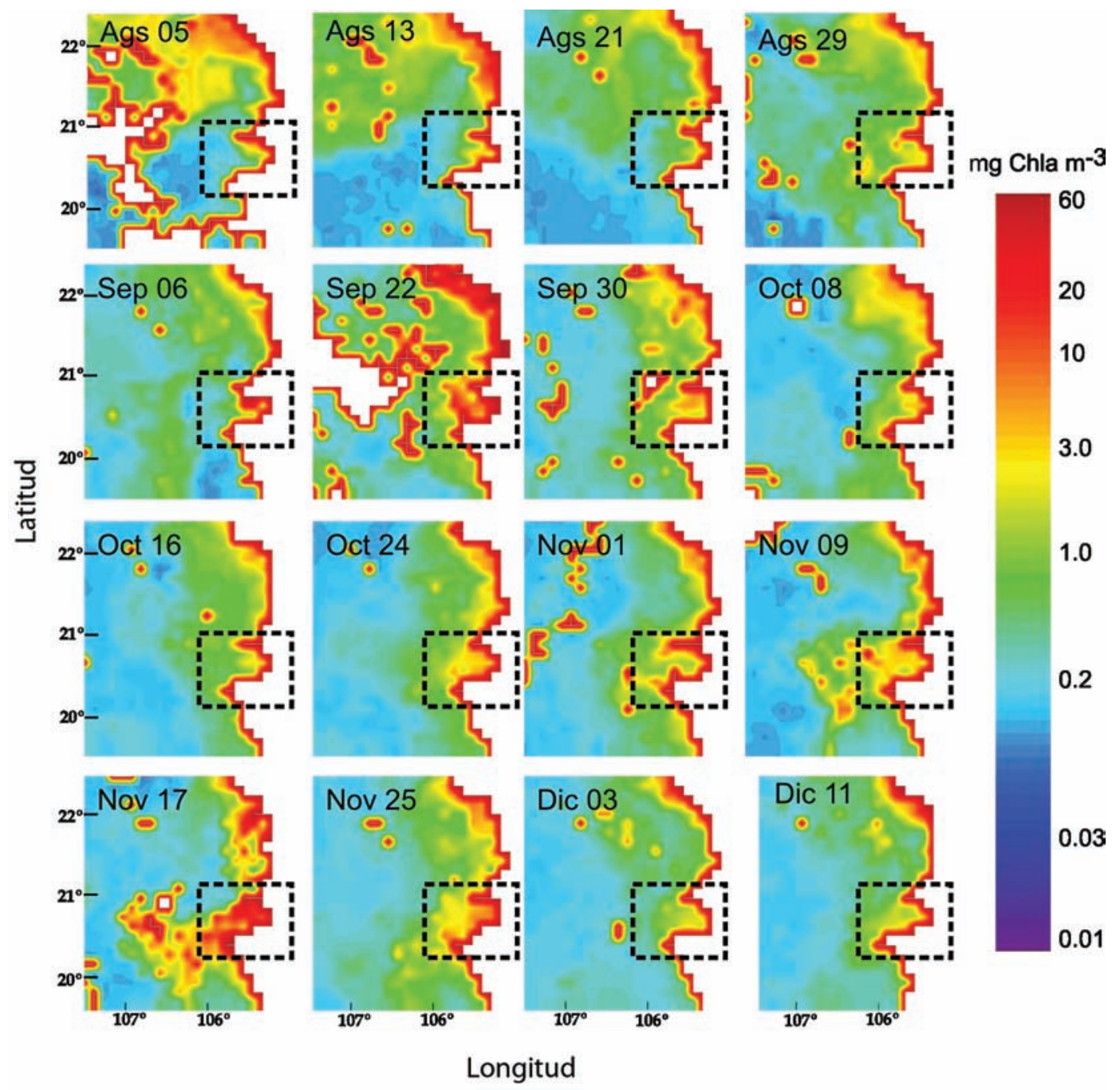

Fig. 4. Compuestos semanales de clorofila satelital dentro de la Bahía de Banderas, para el periodo de julio 30 a diciembre 12 del 2000. Las secciones de color blanco corresponden a continente y/o a nubes. En cada imagen se indica la fecha en que inicia la semana que representa. La escala de color corresponde a la concentración de clorofila superficial determinada por los algoritmos correspondientes al sensor satelital (imágenes nivel 3).

Fig. 4. Weekly compounds of satellite chlorophyll inside Banderas Bay, during the period from July 30 to December 12 , 2000. White sections correspond to continent and/or clouds. In each image the date in which the week began is indicated. The color scale corresponds to the concentration of surface chlorophyll determined by the corresponding algorithms of the satellital sensor (level 3 images). 
la dominancia o al menos la presencia de $C$. polykrikoides. Lo que sí puede afirmarse a partir de los resultados de este trabajo, es que antes de la proliferación de Cochlodinium informada por Cortés-Lara et al. (2004) existió otro FA muy intenso, en Bahía de Banderas.

$\mathrm{Si}$, por el contrario, el FA de abril a junio del 2000 no estuvo asociado a C. polykrikoides, los altos niveles de Clorofila' se pueden atribuir principalmente a una proliferación multiespecífica, dominada por $C h$. affinis, Ch. decipiens, $P$. micans y $D$. californica, como lo indican las muestras tomadas en las cuatro estaciones de estudio. Los efectos esperados de estos eventos en las condiciones del ecosistema de la bahía (abatimiento de nutrientes inorgánicos, aumento en la materia orgánica disuelta y particulada, exclusión competitiva de grupos fitoplanctónicos específicos, etc.) junto con otras condiciones locales no estudiadas (esfuerzo del viento, grandes aportes de ríos, etc.) pudieron haber provocado que a partir de fines de julio existieran las condiciones propicias para la proliferación cuasi monoespecífica de $C$. polykrikoides.

Por otra parte, si bien en sentido estricto no es adecuada la comparación directa entre los conteos celulares de C. polykrikoides y las $\mathrm{As}_{\mathrm{i}}$ dentro de la bahía, al revisar su variación en el tiempo se aprecia una posible asociación entre estas dos variables, que puede atribuirse a la influencia de los pigmentos fotosintéticos de este dinoflagelado en las propiedades ópticas de la superficie marina de la bahía (Figs. 3 y 4). Los análisis de regresión y de varianza aplicados (no presentados) no mostraron que esta asociación fuera estadísticamente significativa ( $\mathrm{p}=0.05)$; sin embargo, sería interesante realizar este tipo de ejercicios con una mayor cantidad de datos y con conteos celulares más representativos procedentes de una red de estaciones ubicadas también en la parte central y en la boca de la bahía.

Cortés-Lara et al. (2004) informaron una extensión máxima de las manchas del FA de Cochlodinium polykrikoides de $9 \mathrm{~km}$ dentro de la bahía. Sin embargo, al revisar las imágenes de compuestos semanales de clorofila satelital para la bahía, correspondientes al periodo de muestreo de julio a diciembre del 2000, se hace evidente que la extensión de la mancha del FA percibida por SeaWiFS abarcó la totalidad de la bahía (1 $\left.407 \mathrm{~km}^{2}\right)$; además, se aprecia claramente cómo se extienden filamentos de clorofila satelital más allá de este cuerpo costero (Fig. 4: Sep. 22, Oct. 24, Nov. 09, Nov. 17 y Nov. 25). La existencia de estaciones en otras zonas de Bahía de Banderas, además de las cuatro aquí usadas, haría factible dos condiciones deseables: 1) la observación directa no estaría confinada a una sección de la bahía, y 2) la señal de radiancia que el sensor satelital detecta podría asociarse con mayor certeza a organismos fitoplanctónicos específicos.

Durante el 2001 se repitió el patrón de acoplamiento durante la primera mitad del año, entre los niveles de clorofila satelital dentro de la bahía, con los detectados por SeaWiFS en las costas de Sinaloa a Jalisco (Fig. 2, ene.jul. 2001). Este patrón sugiere que durante la segunda mitad del ciclo anual los procesos locales tienen mayor efecto en los niveles de clorofila en Bahía de Banderas; mientras que de enero a junio procesos de mayor escala, tales como ondas atrapadas en la costa, el patrón a mesoescala del forzamiento del viento, meandros y giros que afectan a la región costera de Sinaloa a Jalisco (Zamudio et al. 2002; Lavín et al. 2006), pueden ejercer una fuerte influencia en la biomasa fitoplanctónica de la bahía. Para confirmar esta implicación de los patrones dentro y fuera de este cuerpo costero será necesario implementar un plan de muestreo distinto al que se desarrolló para este trabajo y complementar el estudio de los FA en forma interdisciplinaria con aspectos meteorológicos y físicos.

La comparación de las $\mathrm{As}_{\mathrm{i}}$ entre los dos años analizados muestran que los niveles de Clorofila' para el 2001 fueron mucho menores que los detectados por SeaWiFS para el año anterior (Fig. 3). Este hecho concuerda con el decaimiento de las tasas de producción primaria fitoplanctonica para el 2001 respecto al 2000 reportado por Martínez-Gaxiola et al. 2007, situación que puede ser resultado del cambio de una condición "La Niña" a una 
"No-La Niña" en el Pacífico tropical mexicano; es decir, es posible que estos niveles de $\mathrm{As}_{\mathrm{i}}$ se deban a que el sistema está retornando a una condición cercana a la normal, después de haber estado bajo la influencia de los eventos El Niño y La Niña.

Además de las diferencias en las magnitudes de las $\mathrm{As}_{\mathrm{i}}$ entre estos dos años, existe un desfase temporal en la presencia de las máximas anomalías positivas. En el 2001 éstas se presentaron en febrero, junio-agosto, la última semana de septiembre, noviembre y diciembre (Fig. 3, 2001). Los FA de abril a agosto fueron multiespecíficos, sin registros de mortandad de organismos en la bahía, sólo se asocia al cambio de coloración en la superficie marina con una intensidad mucho menor a la reportada para los FA de la segunda mitad del año. Las $\mathrm{As}_{\mathrm{i}}$ positivas de septiembre a diciembre corresponden a una proliferación dominada principalmente por C. polykrikoides, evento asociado de nueva cuenta a una alta mortandad de peces detectada en diferentes regiones costeras de la bahía. El hecho de que durante los dos años analizados se haya presentado un FA por C. polykrikoides en el mismo periodo puede obedecer a la conjunción de: a) condiciones locales prevalecientes sobre las regionales durante las estaciones verano-otoño; b) condiciones ecológicas idóneas provocadas por las proliferaciones previas durante las épocas invierno-primavera.

A partir de lo discutido, resulta evidente la utilidad que representa el añadir a los estudios costeros algún tipo de análisis producto de información derivada de SRS. En particular, al estudiar aspectos relacionados con biología marina, el uso de sensores de color del océano añaden una perspectiva espacio-temporal sinóptica que no puede alcanzarse con los muestreos in situ; en reciprocidad, la información de estos SRS carece de peso sin el apoyo y soporte de estudios en campo y laboratorio. En el caso de la proliferación de $C$. polykrikoides para Bahía de Banderas durante 2000 y 2001, las imágenes de clorofila satelital de SeaWiFS y el análisis de anomalías de Clorofila' demuestran que la extensión del evento fue mucho mayor que la reportada originalmente y que durante la primera mitad de estos años se presentaron en forma previa FA, dos especialmente intensos durante el 2000. Estas proliferaciones durante la primera mitad del 2000 y 2001 fueron multiespecíficas y abren la posibilidad de que tengan un papel determinante en la adecuación de las condiciones del medio para las proliferaciones de $C$. polykrikoides en conjunto con condiciones en la bahía de origen predominantemente local durante la segunda mitad de cada año.

\section{AGRADECIMIENTOS}

Este trabajo se llevó a cabo con los proyectos UR-690 en el 2000, 975.03-P, 901.05-P y 909.06-P. apoyados por la Dirección General de Educación en Ciencia y Tecnología del Mar y el Cosnet respectivamente.

\section{RESUMEN}

Se analizaron las proliferaciones algales ocurridas en Bahía de Banderas, México, durante los años 2000 y 2001 a partir de información derivada del sensor de color del océano SeaWiFS. Estos eventos estuvieron relacionados con los máximos niveles de Anomalias Semanales de Clorofila' $\left(\mathrm{As}_{\mathrm{i}}\right.$; variable construida para analizar la variación temporal de la clorofila dentro de la bahía). Las proliferaciones ocurridas en los periodos invierno-primavera fueron multiespecíficos, mientras que en verano-otoño fueron dominadas por una sola especie. Se plantean dos propuestas: 1) las proliferaciones algales de invierno-primavera están fuertemente relacionadas a procesos de mesoescala dada su aparente sincronía temporal con altos niveles de clorofila en las costas de Sinaloa a Jalisco; 2) mientras que las proliferaciones de Cochlodinium polykrikoides durante los periodos verano-otoño son favorecidas por el efecto de otras proliferaciones algales previas sobre el ecosistema y por condiciones predominantemente locales en la bahía.

Palabras clave: proliferaciones algales, sensores semotos satelitales, SeaWiFS, clorofila, Bahía de Banderas, Cochlodinium polykrikoides.

\section{REFERENCIAS}

Bograd, S. J. \& R. J. Lynn. 2001. Physical-biological coupling in the California Current during the 1997-99 El Niño-La Niña cycle. Geophys. Res. Lett. 28: 275-278. 
Brown, C.W. \& J.A. Yoder. 1994. Coccolithophorid blooms in the global ocean. J. Geophys. Res. 99:7476-7482.

Cannizzaro, J.P., K.L. Carder, F.R. Chen, J.J. Walsh, Z. Lee, C. Heil \& T. Villarreal. 2002. A novel optical classification technique for detection of red tides in the Gulf of Mexico: Application to the 2001-2002 bloom event, p. 43. En Proceedings, Xth International Conference on Harmful Algae, St. Pete Beach, Florida, 21-25 October 2002. Florida Fish and Wildlife Conservation Commission and Intergovernmental Oceanographic Commission of UNESCO.

Carrasco, J.F. \& J. Quintana-Arena. 2004. Description of the atmospheric conditions observed in Chile during 1997-2000 associated to El Niño-La Niña, Southern Oscillation, p. 43/59. En S. Avaria, J. Carrasco \& E. Yáñez (eds). El Niño-La Niña 1997-2000. Sus efectos en Chile. Comité Oceanográfico Nacional, Chile.

Cortés-Lara, M.C., R. Cortés-Altamirano \& A.P. SierraBeltrán. 2004. Presencia de Cochlodinium catenatum (Gymnodiniales: Gymnodiniaceae) en mareas rojas de Bahía de Banderas, Pacífico Mexicano. Rev. Biol. Trop. 52: 35-49.

Cullen, J.J., A.M. Ciotti, A.F. Davis \& M.R. Lewis. 1997. Optical detection and assessment of algal blooms. Limnol. Oceanogr. 42:1223-1229.

Dierssen, H.M., R.M. Kudela, J.P. Ryan y R.C. Zimmerman. 2006. Red and black tides: Quantitative analysis of water-leaving radiance and perceived color for phytoplankton, colored dissolved organic matter, and suspended sediments. Limnol. Oceanogr. 51: 2646-2659.

Fiedler, C. P., F. P. Chavez, D. W. Behringer \& S. B. Reilly. 1992. Physical and biological effects of Los Niños in the eastern tropical Pacific, 1986-1989. Deep-Sea Res. 39:199-219.

Kahru, M. \& B.G. Mitchell. 1998. Spectral reflectance and absorption of a massive red tide off Southern California. J. Geophys. Res. 103: 21601 - 21609.

Lavín, M.F., E. Beier, J. Gómez-Valdés, V.M. Godínez \& J. García. 2006. On the summer poleward coastal current off SW México. Geophys. Res. Lett., 33: L02601.

Martínez-Gaxiola, M.D., R. Sosa-Ávalos y G. GaxiolaCastro. 2007. Producción de carbono orgánico en los mares mexicanos (1998-2005). En: Carbono en ecosistemas acuáticos de México. Gaxiola-Castro, G. y B. Hernández-de-la-Torre (Eds.). Instituto Nacional de Ecología (INE) y Centro de Investigación Científica y Educación Superior de Ensenada (CICESE). México D.F., México.
Roesler, C. S., S. M. Etheridge \& G. C. Pitcher. 2004. Application of an ocean color algal taxa detection model to red tides in the Southern Benguela. En: Steidinger, K. A., Lansdberg, J. H., Tomas, C.R., and Vargo, G. A. (Eds.). Harmful Algae 2002. Florida Fish and Wildlife Conservation Commission, Florida Institute of Oceanography, and Intergovernmental Oceanographic Commission of UNESCO, Paris.

Sathyendranath, S., L. Watts, E.Devred, T. Platt, C. Caverhill y H. Maass.2004. Discrimination of diatoms from other phytoplankton using ocean color data. Mar. Ecol. Prog. Ser. 272: 59-68.

Schoefield, O., J. Grzymski, W.P. Bisset, G.J. Kirkpatrick, D.F. Millie, M. Moline \& C.S. Roesler. 1999. Optical monitoring and forecasting systems form harmful algal blooms: Possibility or pipe dream. J. Phycol. 35:1477-1496.

Sosa-Ávalos, R., M.D. Martínez-Gaxiola \& G. GaxiolaCastro. 2006. Producción primaria en el Pacífico tropical mexicano a partir de datos derivados de sensores remotos. En: Los recursos pesqueros y acuícolas de Jalisco, Colima y Michoacán. JiménezQuiroz, M.C. y E. Espino-Barr (Eds.). Secretaría de Agricultura, Ganadería, Desarrollo Rural, Pesca y Alimentación (SAGARPA) - Instituto Nacional de la Pesca (INP). México, D.F., México.

Steidinger, K.A. \& K. Haddad. 1981. Biologic and hydrographic aspects of red tides. Bioscience 31:814-819.

Stumpf, R.P. 2001. Applications of Satellite Ocean Color Sensor for Monitoring and Predicting Harmful Algal Blooms. Hum. Ecol. Risk Assess. 7:1363-1368.

Subramaniam, A., C.W. Brown, R.R. Hood, E.J. Carpenter \& D.G. Capone. 2002. Detecting Trichodesmium blooms in SeaWiFS imagery. Deep-Sea Res. 49: 107-121.

Subramaniam, A., E.J. Carpenter \& P.G. Falkowski. 1999. Biooptical properties of the marine diazotrophic cyanobacteria Trichodesmium spp.: II. A reflectance model for remote sensing. Limnol. Oceanogr. 44:618-627.

Tester, P.A., R.P. Stumpf \& K. Steidinger. 1998. Ocean color imagery: What is the minimum detection level for Gymnodinium breve blooms?, p. 149-151. En B. Reguera, J. Blanco, M.L. Fernandez \& T. Wyatt (eds). Harmful algae. Xunta de Galacia and Intergovernmental Oceanographic Commission of UNESCO, Paris.

Tomlinson, M.C., R.P. Stumpf, V. Ransibrahmanakul, E.W. Truby, G.J. Kirkpatrick, B.A. Pederson, G.A. Vargo \& C.A. Heil. 2004. Evaluation of the use of SeaWiFS imagery for detecting Karenia brevis harmful algal 
blooms in the eastern Gulf of Mexico. Remote Sens. Environ. 91:293-303.

Utermöhl, H. 1958. Zur Vervollkomnug der kuantitativen phytoplankton methodik. Mitt. Int. Ver. Limnol. 9:1-38.
Zamudio, L., H.E. Hurlburt, E.J. Metzger \& O.M. Smedstad. 2002. On th evolution of castally trapped waves generated by Hurricane Juliette along the Mexican West Coast. Geophys. Res. Let. 29: 2141 . 